\title{
La Adaptación de \\ Materias del Ámbito \\ Jurídico-Social al \\ Crédito Europeo. La \\ Propuesta de las \\ Universidades de \\ Galicia
}

\section{$M^{\mathrm{a}}$ Esther Martínez Figueira ${ }^{\mathrm{a}}$}

\author{
Rebut: 07/05/2010 Acceptat: 10/06/2010
}

\section{Resumen}

En este artículo se pretende destacar la labor realizada por la ACSUG en materia de convergencia europea por ser nuestra agencia de referencia. Si a esto unimos el hecho de haber sacado a luz una serie de publicaciones sobre el proceso de adaptación de materias de algunas titulaciones universitarias gallegas al crédito europeo, es lógico comprender el no querer permanecer impasibles a este fenómeno de divulgación de conocimientos. Así, nos sentimos obligados moralmente de hacérselo saber al lector de que desde la ACSUG tiene a su disposición una serie de publicaciones que pueden servir de referente en este proceso de cambio del que participamos. Por ello, en este trabajo se presentan a la comunidad universitaria algunas de las publicaciones del proyecto ACSUG sobre el proceso seguido y resultados obtenidos en la adaptación de materias de algunas titulaciones del ámbito jurídico-social del Sistema Universitario de Galicia (SUG).

Palabras clave: ECTS, proyecto ACSUG, titulaciones jurídico-sociales

\section{Adaptation of Legal-Social Subjects to EEES}

\section{Abstract}

This article aims to highlight the work done by the ACSUG in the field of European convergence for its being our reference agency. If we add to this the fact that, a series of publications on the process of adapting some University subjects to European

\footnotetext{
a Universidad de Vigo
} 
credit has been made public, then it is reasonable understanding the refusal to stand still before this phenomenon of spreading the knowledge. Thus, we feel morally compelled to let readers know that they have at their disposal since the ACSUG a number of publications that can be used as a reference point in this process of change which we are part of. Therefore, we present here to the University Community some of the publications of the ACSUG project about the process and results in the adaptation of some legal-social subjects of the University System of Galicia (SUG).

Key words: ECTS, ACSUG project, legal-social qualifications

\section{Introducción}

Como todos los grandes cambios, el Espacio Europeo de Educación Superior (EEES) cuenta con una historia que, aunque breve, ha supuesto todo un cambio tanto en la mentalidad como en las estructuras de la universidad, por ejemplo, cambios que afectan a la actual estructuración de los mapas de las titulaciones y planes de estudios, al concepto de calidad universitaria, a la aparición de créditos ECTS, a la redefinición de metodologías, etc. (García, 2009; European Commission, 2010; Colás y De Pablos 2005; Benito y Cruz, 2005).

En este contexto de cambio y búsqueda de innovaciones didácticas y evaluativas, emergen plataformas mundiales para el intercambio de ideas innovadoras que ayuden a evaluar los progresos, analizar las dinámicas emergentes y sus implicaciones políticas y recomendar acciones concretas que permitan cumplir los objetivos de desarrollo nacionales, como es el caso de CMES (2009) ${ }^{1}$; así como experiencias piloto de adaptación de materias sueltas o cursos completos de titulaciones. En todo este recorrido, diferentes agentes de la facultad (alumnado, equipo decanal, personal de bibliotecas, personal de administración y servicios, profesorado...) vienen participando y reconociendo a su vez cierto interés y preocupación por conocer las características del espacio europeo y sus incidencias (Martínez, 2008) en la titulación de la que participan. Particularmente, es digno de destacar el papel que han venido desarrollando, tanto de la Agencia Nacional de Evaluación de la Calidad y la Acreditación (ANECA) como de las diferentes agencias autonómicas (en nuestro caso, la Agencia para la Calidad del Sistema Universitario de Galicia, también denominada ACSUG), para potenciar la mejora de la actividad docente, investigadora y de gestión de las universidades; contribuir a la medición del rendimiento de la educación superior conforme a procedimientos, objetivos y procesos transparentes; proporcionar a las Administraciones Públicas información adecuada para la toma de decisiones; así como informar a la sociedad sobre el cumplimiento de objetivos en las actividades de las universidades ${ }^{2}$.

Particularmente, la ACSUG y según figura en su página web (http://www.acsug.es/galego/webs/objetivos.php), nace con la voluntad de actuar como un servicio a la sociedad y tiene como objetivos la evaluación del Sistema Universitario de Galicia para proponer medidas de mejora de la calidad de los 
servicios que presta éste, especialmente aquellos referidos a la enseñanza, investigación, gestión, servicios y evaluación del profesorado. Así pues, para cumplir este gran objetivo, la ACSUG prevé una serie de actuaciones en materia de espacio europeo, por ejemplo, promover la coordinación entre las universidades gallegas en materia de calidad; efectuar evaluaciones específicas de la calidad de la enseñanza en relación con la formación inicial o permanente impartida por su profesorado; realizar evaluaciones de la calidad de la investigación que se efectúa y valorar su incidencia; analizar los planes de estudio universitarios y evaluar el impacto y los resultados de sus reformas y modificaciones así como de su grado de experimentación; etc.

Al hacer efectivas las actuaciones citadas, ambas agencias promueven su difusión a través de publicaciones que son accesibles al público desde su respectiva página web, bien en formato digital o bien en formato impreso. Entre la variedad de informes y publicaciones que desde su página web se dan a conocer, y que en este texto nos centraremos única y exclusivamente en las que pertenecen a la ACSUG, señalar que éstas se agrupan a priori por temáticas. Así, la ACSUG presenta las mismas según sean sobre la creación y estatutos de la agencia; la evaluación institucional; postgrados; la adaptación de materias al crédito europeo; la inserción laboral de los titulados; así como otras publicaciones referidas a memorias de actividades, análisis de costes de calidad, etc. Particularmente, en este trabajo interesan las publicaciones sobre la adaptación de materias de titulaciones del ámbito jurídico-social.

Así pues, el objetivo de este trabajo es presentar a la comunidad universitaria la síntesis realizada de las publicaciones del proyecto ACSUG sobre el proceso seguido y resultados obtenidos en la adaptación de materias de algunas titulaciones del ámbito jurídico-social del Sistema Universitario de Galicia (SUG). De este modo, el lector podrá hacerse una idea clara y precisa de estos volúmenes que encierran experiencias vividas en despachos y aulas universitarias gallegas $\mathrm{y}$, sobre todo, comunican diseños, desarrollos y evaluaciones de experiencias piloto.

El esquema que se seguirá para la recensión de las obras será por titulaciones y, en cada una de ellas, primero se hará una presentación de la publicación especificando su autoría, la Universidad a la que pertenecen, la referencia bibliográfica y la ficha extraída de la web de la ACSUG; y en segundo lugar, se especificarán los contenidos abordados en cada publicación.

\section{Publicaciones referidas a la titulación de Licenciado en Ciencias de la Actividad Física y del Deporte}

En lo que respecta a la licenciatura de Ciencias de la Actividad Física y del Deporte, la ACSUG nos presenta el trabajo realizado por un grupo de profesores de la Universidad de Vigo. La estructura que siguen es la siguiente ${ }^{3}$.

A través de la coordinación de diez profesores de la licenciatura, se estructura el libro que contiene siete puntos. 
El primer punto, la introducción, sirve para contextualizar el trabajo que engloba la publicación. Así, se recuerda cómo han sido los inicios de la titulación así como también los motivos que llevaron a incorporar ésta al EEES.

El segundo apartado, diseño del proyecto de adaptación, explica cómo ha sido este proceso de adaptación. Así, se incluye en sus páginas los objetivos acordados en la primera reunión del grupo de trabajo que atañen al profesorado (un total de ocho), al alumnado (sólo uno) y a la docencia (dos). También se alude a cómo se hizo la selección de materias participantes en el proyecto, especificando el curso al que pertenecen.

El desarrollo de la experiencia en términos generales, es el tercer aspecto que se aborda en el libro. En este punto se describe someramente cómo se ha iniciado la labor, empezando ésta con la elaboración de las guías docentes. Tras la elaboración de éstas, el grupo de trabajo hizo una búsqueda de sistemas de recogida de información del alumnado, proponiendo una ficha básica para todo el profesorado implicado. Simultáneamente con la elaboración de guías y registro de las mismas, se realizaban actividades informativas y formativas.

Una vez descrito el proceso, el cuarto apartado del libro incluye, a modo de ejemplo, los datos de nueve materias de las que el profesorado facilitó información. Éstas son "Actividades aeróbicas con soporte musical", "Alto rendimiento en fútbol I", "Alto rendimiento en gimnasia aeróbica I", "Deportes de adversario con fines recreativos", "Didáctica de educación física I", "Expresión corporal", "Fundamentos y contenidos didácticos de la natación y actividades acuáticas", "Métodos estadísticos" y "Prevención de lesiones en la práctica deportiva".

En cada una de las anteriores, los datos que se aportan hacen referencia a la descripción de la materia en el plan de estudios, objetivos y carga docente, evolución previa de la materia; el diseño de la adaptación: nuevas metodologías docentes; la adaptación al ECTS; los métodos de evaluación; los resultados académicos y encuestas de esfuerzo y opinión del alumnado.

En quinto punto, sintetiza la evaluación global del proceso de experimentación desde la perspectiva de cinco informantes:

1. El equipo decanal destaca, entre otras cosas, que aún queda un largo camino por recorrer no sólo por los órganos de gestión de las universidades gallegas sino también por toda la comunidad universitaria tanto PDI, PAS y alumnado.

2. Los profesores implicados, realizan una opinión sobre tres aspectos:

Uno, en cuanto a la organización de los centros. En este caso, se destaca, por ejemplo, que la infraestructura es fundamental para la realización de tutorías colectivas.

Dos, en cuanto a cuestiones docentes y académicas, en concreto, sobre la dimensión práctica del curriculum en relación a la proporción de créditos de 
aula/laboratorio, a la proporción de contenidos procedimentales/conceptuales y a la proporción de métodos activos/expositivos; sobre la planificación de la docencia; sobre la metodología y recursos empleados destacando las activas y los más vinculados a las nuevas tecnologías, respectivamente; sobre la función tutorial en la docencia universitaria; $y$, finalmente, acerca de los mecanismos de evaluación del aprendizaje y reconsideración de la propia evaluación, siendo conscientes de la tarea pendiente que es aún la evaluación continua.

Tres, en relación a la experimentación, los profesores opinan sobre la aplicación de las guías docentes, en cuanto a su elaboración, su dificultad, los aspectos positivos que éstas encierran o los aspectos negativos que le reconocen; la evaluación del proceso respecto al profesorado, al alumnado y a la docencia impartida; el grado de satisfacción con la experiencia, del que me atrevo a adelantar que se reconoce que la experiencia ha resultado interesante en cuanto que el profesorado se ha visto en la necesidad de revisar sus planes de estudio y reconsiderar la forma de impartir la docencia; y la necesidad de replantear concepciones educativas o la manera de entender la enseñanza universitaria.

3. Profesores no implicados en la experiencia (en total 8), esto es, se recoge la opinión sobre los aspectos positivos, los negativos y los que se deberían de tener en cuenta.

4. El alumnado, también se recogen opiniones de este colectivo, donde se puede observar la diversidad de las mismas.

5. El PAS, los autores del libro incluyen este grupo de profesionales pero indicando que no se recogen opiniones de los mismos ya que estos no han manifestado opinión alguna.

En el sexto apartado de la publicación, se sugieren una serie de recomendaciones sobre las necesidades sentidas a nivel de organización, de recursos y de gestión.

El libro termina con un séptimo punto en el que se incluyen apéndices electrónicos. Por ejemplo, presentaciones empleadas en el proceso de implantación y difusión de la experiencia, guías docentes más significativas de las materias adaptadas, cuadros de estimación de esfuerzo en créditos ECTS, fichas para la recogida de información del alumnado y el día del examen, encuestas para el profesorado que participó en la experiencia, modelos de cartas enviadas a miembros de la comunidad universitaria para invitarlos a participar, etc.

\section{Publicaciones referidas a la titulación de Licenciado en Psicología}

Entre los materiales publicados sobre la titulación de Psicología, la ACSUG presenta dos volúmenes. Uno, referido a la primera y segunda experiencias piloto 
desarrolladas en los cursos 03-04 y 04-05/05-06 en la titulación citada de la Universidad de Santiago desde el punto de vista del profesorado; otro, centrado sólo en la segunda experiencia pero desde la perspectiva del alumnado de la misma titulación. Pasamos pues a describir cada uno de estos dos ejemplares.

\subsection{Primera y segunda experiencias piloto: perspectiva profesorado}

Tal como decíamos, uno de los ejemplares hace referencia a las $1^{\mathrm{a}}$ y $2^{\mathrm{a}}$ experiencias piloto.

Con esta publicación se pretende dar a conocer a la comunidad universitaria del objetivo que la titulación y facultad de Psicología de la Universidad de Santiago viene a desarrollar mediante diferentes iniciativas para enfrontar el proceso de convergencia europea. Así, se persigue adaptar la titulación a las condiciones necesarias para garantizar cierta competitividad en el nuevo contexto que, al hilo del proceso de convergencia, se establezca. Bajo este objetivo, la publicación de la que hablamos presenta siete apartados claramente diferenciados y que se describen a continuación.

En primer lugar, el lector se puede encontrar con una introducción en la que se especifica el objetivo de la titulación, la situación de la titulación dentro del SUG, el origen del proyecto así como los objetivos de la experiencia piloto de adaptación de materias al crédito europeo en la facultad de Psicología de la Universidad de Santiago en los años académicos 04-05 y 05-06.

En un segundo momento, se describe el diseño del proyecto de adaptación en aquellas materias impartidas por profesores que en su momento manifestaron su interés por participar. Se reconoce en este apartado del reto al que se está a someter al profesorado ya que las condiciones actuales en que imparten su docencia están lejos de ser las más adecuadas.

El tercer punto de la publicación, comienza haciendo referencia a cómo se ha desarrollado la primera experiencia iniciada en el curso 2003-04 a través de un programa de formación permanente que incluía seminarios sobre herramientas didácticas y experiencias relacionadas con el proceso de convergencia europea que se estaban a desarrollar en otras universidades. Por otra parte, los profesores interesados en participar en estas experiencia piloto, fueron orientados en la elaboración de guías docentes para implantar en la misma.

Una vez descrita ésta, los autores se refieren a la segunda experiencia que, tal como ya se ha indicado, se ha puesto en marcha en los cursos 2004-05 y 2005-06. En esta descripción de acciones llevadas a cabo, se recuerda cómo ha sido su inicio, las intenciones de sus responsables, el cumplimiento de las guías, el proceso de incorporación de materias, etc.

En el cuarto punto de la publicación, se presentan los objetivos y datos de evolución previa de las calificaciones en cada materia participante; para pasar a indicar los datos 
generales del desarrollo de las experiencias basados en el esfuerzo y opinión del alumnado participante en las materias de "Atención y emoción", "Psicología fisiológica", "Técnicas de modificación de conducta", "Psicofisiológica aplicada", "Psicología del currículo", "Psicología de la lectura", "Psicología del lenguaje", "Familia y desarrollo humano", "Dificultades en el desarrollo del lenguaje e intervención", "Rehabilitación psicosocial", "Construcción de los conocimientos y de los aprendizajes escolares: dificultades e intervención", "Orientación Vocacional", "Toma de decisión vocacional e inserción socio-laboral" y "Evaluación e intervención en Psicología de la Salud".

En un quinto apartado, se incluye la evaluación global del proceso de experimentación, es decir, el resultado crítico de las experiencias de adaptación al crédito europeo, recogiéndose tanto las consideraciones del equipo decanal como las opiniones proporcionadas por los diferentes agentes implicados. Así, algunos de los aspectos que se destacan en este punto hacen referencia a destacar la alta disposición del profesorado a participar; el que se hayan confirmado las previsiones; el que, con un tamaño y un número de grupos adecuado, la experiencia es fácilmente generalizable; el que se haya puesto de manifiesto que el alumnado es inicialmente remiso a las propuestas de cambio metodológico que supongan un mayor protagonismo en las actividades de enseñanza-aprendizaje, etc; o el que la coordinación entre materias haya sido un aspecto problemático o complejo.

El sexto apartado de la publicación sintetiza una serie de recomendaciones a tener en cuenta sobre las necesidades de organización, de recursos y de gestión para incentivar la actualización y la mejora de la actividad docente. Algunas de éstas se sitúan en temáticas sobre ratios alumno-profesor, cómputos de dedicación docente, horarios, coordinación de materias, etc.

Finalmente, el informe termina incluyendo una serie de anexos a modo informativo y de consulta. Así, los dos primeros son los cuestionarios de evaluación de la experiencia piloto que se han empleado (uno para el alumnado, otro para el profesorado); el tercer anexo sintetiza las metodologías docentes consignadas; y el cuarto anexo incluye la relación del personal implicado en este trabajo (profesorado y equipo decanal). En definitiva, esta amplia documentación anexionada al final del libro es muy válida para profundizar en la experiencia realizada.

\subsection{Segunda experiencia piloto: perspectiva alumnado}

El segundo ejemplar que se indicaba al comienzo de este bloque, recoge de nuevo la $2^{a}$ experiencia piloto en términos de ECTS en la licenciatura de Psicología en la USC pero centrando la experiencia desde el punto de vista del alumnado ${ }^{4}$.

Este volumen sintetiza la segunda acción realizada en la titulación de Psicología de la USC en adaptación de créditos europeos. Tal como se ha señalado, en este caso, escuchando la voz del alumnado y observando el trabajo de los y las estudiantes de 
la citada titulación en términos ECTS, pasamos a relacionar los puntos que se abordan. En total son 9 apartados que detallamos.

En primer lugar, los autores realizan una contextualización teórica del tema que nos ocupa: el Espacio Europeo de Educación Superior y el crédito europeo. Así, realizan una referencia teórica a los inicios del proceso de convergencia europea, a sus finalidades, a responsabilidades de los partícipes del proceso de adaptación de créditos, etc.

En segundo lugar, se realiza una reseña histórica de cómo ha sido el inicio de la Facultad de Psicología de la USC en el proceso de convergencia europea. Se explica someramente el tipo de acciones y actividades que se comienzan a realizar en esta línea y particularmente las relacionadas con el estudio del que se informa en esta publicación. Por último, este apartado termina indicando las actividades formativas que desde la Facultad se han realizado de acuerdo a la demanda surgida en materia de ECTS.

En tercer lugar, se expone la justificación del estudio realizado que, sintetizando este tercer apartado, se puede decir que éste nace tras la situación de incertidumbre que hay en algunas universidades y facultades españolas así como en la de Santiago. Por lo tanto, en este informe se ha tratado de analizar la carga de trabajo de las actuales materias con la intención de tener algún referente en el proceso de adaptación al crédito europeo. Así, este informe da cuenta de ello.

En el cuarto apartado, se recoge el objetivo general que, tal como venimos diciendo, consiste en estimar el esfuerzo necesario para superar las distintas materias de la titulación en términos de horas de trabajo. Para el alcance de éste, los autores desglosan este objetivo en otros 6 .

En función de los objetivos del estudio, en el quinto apartado se describe la metodología adoptada. Así, se alude a los sujetos informantes, señalando los indicadores que se han tenido en cuenta para su selección (continuidad en los cursos, calificaciones, etc); y al procedimiento seguido con los estudiantes para recabar las informaciones oportunas.

Los datos recogidos en el presente estudio se sintetizan en el sexto punto de la publicación. Estos permiten enfocar el trabajo del alumnado en los siguientes niveles según sea:

- El trabajo total y el trabajo no presencial a lo largo de los cursos, esto es, el trabajo que a lo largo de los cursos de la titulación exigen las tareas académicas en su globalidad, para todo el alumnado de la muestra y para aquel con calificación igual o superior a la media.

- El trabajo total de las materias en cada uno de los cursos de la titulación, es decir, se analiza la carga de trabajo total que para el alumnado implica la asistencia a las aulas y las demás tareas académicas, en las distintas materias troncales y obligatorias. 
- El trabajo no presencial de las materias en cada uno de los cursos de la titulación, es decir, se analiza la carga de trabajo no presencial por crédito de las materias troncales y obligatorias por separado.

- El trabajo exigido por cada tarea no presencial a lo largo de los cursos, esto es, se analiza la carga de trabajo de cada tipo de tarea académica no presencial en los distintos cursos de la titulación.

- El trabajo exigido por cada tarea en las materias en cada uno de los cursos de la titulación.

- El tiempo medio dedicado a cada actividad, es decir, se presentan los datos obtenidos sobre el tiempo que demandan, por unidad, las distintas tareas.

El séptimo apartado de la publicación, informa sobre la valoración realizada sobre la correspondencia entre el actual modelo de enseñanza-aprendizaje y el modelo europeo para ofrecer las orientaciones oportunas con el fin de que sirvan para el diseño de los futuros planes de estudio y programaciones docentes. En resumen, en este apartado se hacen públicas las implicaciones que posee el presente estudio para extraer una serie de conclusiones.

En el octavo apartado, se realiza una valoración del trabajo realizado señalando las limitaciones y propuestas que se pueden sugerir para continuar con este estudio.

Finalmente, el informe termina incluyendo una relación de anexos sobre las actividades formativas que han tenido lugar en la Facultad de Psicología de la Universidad de Santiago durante el curso 2004-05; las materias participantes en la experiencia piloto de adaptación al crédito europeo; y una ficha de autorregistro.

\section{Publicaciones referidas a la titulación de Diplomado en Educación Infantil}

En cuanto a los materiales publicados sobre la titulación de Educación Infantil, la ACSUG presenta cuatro volúmenes. Estos son:

\subsection{Titulación de Educación Infantil de Lugo}

El profesorado de la Escuela Universitaria de Formación de Profesorado del Campus de Lugo (Universidad de Santiago) participa en la adaptación de materias a créditos ECTS. Producto de ello es el siguiente informe.

Con esta publicación se pretende dar a conocer a la comunidad universitaria del papel que el profesorado de la E.U. de Formación de Profesorado de Lugo viene desarrollando. Así diremos que el ejemplar se estructura en dos grandes bloques temáticos. El primero que se refiere a la presentación del informe del curso 2005-06 
tras la reunión del grupo de profesorado implicado; y el segundo que comprende dos guías docentes. Veamos cada uno por separado.

El primer apartado de la publicación, tal como decíamos, responde al informe del curso 2005-06 que presenta el profesorado voluntario y que está adscrito al programa de experimentación y puesta en marcha de manera adelantada y de forma piloto de los créditos ECTS en la titulación de Educación Infantil. Éste tiene por finalidad informar de las actividades que desarrolla dicho profesorado.

En sus primeras páginas se indica el procedimiento que se ha seguido para la recogida de la información. Así, uno de los recursos utilizados para recabar datos ha sido el informe personal de cada docente con sus respectivas observaciones. Así, aunque estos documentos a los que nos estamos refiriendo se incluyen en forma de anexos, decir que responden a las siguientes materias: "Historia de Galicia.", "Sistemas contemporáneos de la Educación Infantil", "Conocimiento del medio social y cultural", "Ética para educadores", "Conceptos fundamentales de didáctica", "Expresión musical y su didáctica", "Sociología de la educación" y "Teorías e instituciones contemporáneas de la educación".

Cada uno de estos informes se presentan en base a los objetivos previstos, las actividades realizadas en el marco ECTS, las dificultades encontradas y las necesidades sentidas.

Otros recursos utilizados para recabar información han sido las observaciones, el desarrollo del programa de competencias y el informe anterior en el que se adelantaban algunas dificultades.

Además de contemplar los recursos utilizados, los autores continúan este apartado mencionando muy minuciosamente los pasos dados y terminan presentando las conclusiones generales a las que llegaron y que han de ser tenidas en cuenta en la mayor brevedad posible.

El segundo apartado de la publicación comprende las guías docentes de dos asignaturas que participaron en el proyecto piloto de adaptación. Éstas son la de "Música de Educación Infantil" y la de "Expresión musical y su didáctica" y los elementos que se abordan en cada una de ellas son: datos descriptivos del curso/materia, sentido del curso en el plano de formación del profesorado universitario, objetivos/competencias generales, contenidos de la materia, orientaciones metodológicas y distribución de ECTS y orientaciones de evaluación.

\subsection{Titulación de Educación Infantil de Ourense}

En cuanto a Educación Infantil que se imparte en el campus de Ourense, la ACSUG nos presenta el trabajo realizado por un grupo de profesores de la Universidad de Vigo, en colaboración con personal técnico de la agencia ${ }^{5}$. 
Este volumen presenta un formato diferente a los que venimos describiendo. Sin ser mejor ni peor, sino distinto, la publicación encierra una interesante variedad de trabajos realizados por el grupo de profesores que imparten en la Facultad de Ciencias de Educación del campus de Ourense. Con todo ello, describimos cada uno de los diez puntos que engloba que, al tener una autoría propia de un solo profesor o grupos pequeños de trabajo, hace que los presentemos de manera diferenciada cada uno de ellos.

1. Resultados y evaluación de la experiencia de implantación de EEES en Educación Infantil-Ourense. Aspectos introductorios. El autor de este primer punto del manual inicia su aportación recordando los objetivos de la titulación, la situación de la misma dentro del SUG, el origen del proyecto así como los motivos de la participación del grupo de trabajo. Particularmente, se indica que el proyecto es visto como un paso más para ir introduciendo la cultura de la calidad, la evaluación y la mejora permanente. Por otro lado, también recuerda el diseño del proyecto de adaptación tanto en cómo ha sido la selección inicial de las materias piloto como finalmente cuáles han sido las materias que realmente han desarrollado la experiencia.

2. Desarrollo de la experiencia. Un grupo de cuatro profesores de la Facultad, inician esta segunda aportación realizando un resumen de los principales elementos del proyecto para dar paso a la presentación de un modelo de guía docente de la materia "Teorías y sistemas contemporáneos de la educación". En esta guía observamos información sobre la contextualización de la materia, los objetivos, prerrequisitos, bloques y temas de contenido, metodología y estrategias de enseñanza-aprendizaje, plan de trabajo del alumnado con especificación aproximada del tiempo de aprendizaje, bibliografía y otros materiales, evaluación de los procesos y resultados de aprendizaje (criterios de evaluación) y evaluación del proceso docente. Esta aportación termina realizando una síntesis sobre los métodos docentes y sistemas de evaluación propuestos, el cumplimiento de las guías y la incorporación de nuevas materias proyecto.

3. Adaptación de la materia "Psicología de la educación y del desarrollo en la edad escolar" al sistema de transferencia de créditos europeos (ECTS en la Universidad de Vigo). La autora de esta aportación describe la materia de "Psicología de la educación y del desarrollo en la edad escolar" de acuerdo con el plan de estudios vigente. Posteriormente, presenta el diseño para la adaptación haciendo hincapié en los cambios en que nos vemos sometidos (en el modelo de aprendizaje, en el modelo de enseñanza, en la forma de trabajar, en la evaluación, en la utilización de las tecnologías de la información y la comunicación y en el uso de la biblioteca). En esta presentación del diseño para la adaptación, también incluye las competencias académicas y profesionales que incluye en la guía docente de la materia que se está experimentando (instrumentales, interpersonales y sistémicas) así como las distintas metodologías de enseñanza que ha tenido 
en cuenta a la hora de impartir la materia (clases presenciales, clases no presenciales y tutorías) para que los alumnos adquieran las competencias establecidas.

En otro momento y a continuación del punto anterior, la autora se refiere a la cuantificación del trabajo de los alumnos, a los métodos de evaluación y a las calificaciones de los mismos,

Por último, se presenta una evaluación de la experiencia, lo positivo y lo negativo, así como también se presenta una propuesta de mejora para cursos posteriores.

4. Valoración inicial de la adaptación de la materia "Historia de la cultura" a los créditos ECTS en la titulación de Educación Infantil. En este caso, el autor de la materia "Historia de la Cultura", presenta su particular valoración del diseño de experimentación de dicha materia. Siendo así, realiza en primer lugar una contextualización de la asignatura aludiendo a su situación en el plan de estudios vigente; para pasar entonces a explicar el diseño de la adaptación a créditos ECTS. En este punto, se citan los objetivos que orientan el mismo (en total 6), las competencias que están vinculadas a los resultados de aprendizaje esperados (de carácter general, instrumentales, interpersonales y sistémicas) así como la metodología docente empleada que, en este caso, estaba basada en la existencia de todo un conjunto de principios de procedimiento o de actuación docente. De entre estos destacar el establecimiento de un contexto favorable para las discusiones en el aula, la transformación del papel docente, entre otros. A partir de dichos principios docentes, la docencia del profesor en esta materia se distribuye en dos grandes núcleos: las clases y las tutorías.

Continúa su aportación realizando la cuantificación del trabajo del alumnado, señalando los métodos de evaluación, presentando las encuestas utilizadas para valorar el esfuerzo y conocer la opinión del alumnado implicado; para así terminar el texto haciendo una valoración de la experiencia y las perspectivas de futuro.

5. Resultados y evaluación de la experiencia de implantación del espacio europeo de educación superior en la materia de "Introducción a la geografía". Al igual que en capítulos anteriores, la profesora de la asignatura "Introducción a la geografía", comienza su aportación haciendo una descripción de la situación de su materia en el plan de estudios de la titulación, señala los objetivos generales así como los transversales a conseguir, indica el número de alumnos matriculados, alude al rendimiento académico de estos, se refiere a la entrega de un CD que almacenaba los contenidos básicos de la materia, las prácticas con el material preciso para realizarlas, las páginas web de referencia, la cartografía y las diapositivas más destacadas; y que actuaba dicho CD como instrumento de las nuevas metodologías adoptadas en la materia. 
A continuación de este punto, la autora especifica la cuantificación del trabajo del alumnado que participa en la experimentación de la materia, los criterios asumidos y que han sido aplicados, los métodos de evaluación tenidos en cuenta, su opinión de los resultados académicos analizados globalmente así como por actividades, las encuestas utilizadas para conocer el grado de esfuerzo y opinión de los alumnos en cuanto a la carga de trabajo, el grado de satisfacción y los problemas encontrados desde su punto de vista.

Termina este capítulo indicando su valoración de la experiencia, la cual ha sido satisfactoria; y la perspectiva de futuro, donde alude al posible exceso de trabajo que tendrán los alumnos de la titulación cuando todas las materias estén adaptadas a la convergencia europea.

6. Valoración inicial de la adaptación de la materia de "Nuevas tecnologías aplicadas a la educación" a los créditos ECTS en la titulación de Educación Infantil. Las dos profesoras que imparten "Nuevas tecnologías aplicadas a la educación" comienzan su aportación a la publicación recordando los inicios que tuvo la experimentación. Con estos antecedentes, justifican el sentido de su capítulo.

Al igual que en otras materias, las autoras ubican la materia en el plan de estudios, justifican a través de 7 objetivos el diseño de la adaptación de "Nuevas tecnologías aplicadas a la educación" a créditos ECTS, especifican el contexto donde se realiza dicha adaptación a través de 17 competencias, recordando los hándicaps con que se encontraron en este momento de adaptación y la incidencia que tiene las Tecnologías de la Información y la Comunicación (TICs) para los titulados en educación; señalan la metodología docente que implementan (activa y participativa) y los principios en que se basan, concretándose y combinándose con cinco estrategias metodológicas fundamentales (enseñanza presencial, formación on-line, trabajo individual, trabajo en grupo y tutorías). Continúan cuantificando el trabajo del alumnado y especificando la distribución de horas por actividades realizadas; señalando los criterios de evaluación de carácter general y específico que se siguen; valorando los resultados académicos de los alumnos desde una perspectiva global como por materias, comunicando los resultados obtenidos en las encuestas de esfuerzo y de opinión que al término del cuadrimestre le pasaron a su alumnado para interesarse por el trabajo autónomo desarrollado así como por la satisfacción con la experimentación realizada. Así, los contenidos que presentan y discuten versan sobre el trabajo autónomo, la utilización de las tutorías, la plataforma de teleformación, entre otros.

Terminan su aportación realizando una valoración de lo realizado, con sus puntos fuertes y sus puntos débiles; y las perspectivas de futuro que tiene su experiencia piloto. 
7. El portafolio como instrumento para la evaluación de la adaptación a los créditos ECTS en las prácticas de laboratorio de la materia de "Nuevas tecnologías aplicadas a la educación". En el contexto de experimentación de la adaptación de créditos ECTS de dicha materia, las mismas autoras inciden ahora en la relevancia que posee la parte práctica de la materia, tanto por tener una carga docente de 3 créditos (en relación a la materia total, es de dos tercios de la misma) como por su sentido aplicativo y práctico, procedimental y competencial.

Así pues, las autoras proceden a describir someramente un aspecto vinculado a las prácticas de la asignatura: el portafolio, como instrumento para su evaluación, ofreciendo información sobre los contextos de uso y algunas reflexiones de los estudiantes y docentes sobre la pertinencia y validez del mismo.

Por todo ello, se inicia este punto aludiendo al contexto curricular e institucional de las prácticas de laboratorio de la citada asignatura así como a su contexto académico.

En segundo lugar, se justifica el papel que desempeña el instrumento del portafolio en la evaluación de la implementación, explicando sus características y funciones.

En tercer lugar, indican los resultados de la evaluación de las prácticas de laboratorio con el portafolio enfocando éste desde una doble vertiente: desde la perspectiva del alumnado y desde la perspectiva del profesorado. Así, la del alumnado la realizan sobre la base de sus propias manifestaciones, recogidas en el portafolio y las opiniones recogidas en la encuesta de valoración general de la experimentación a créditos ECTS de la materia. En cuanto a la perspectiva del profesorado, la realizan en base al análisis de los trabajos presentados por los alumnos como resultado del proceso desarrollado en las sesiones prácticas y que se convierten en ejes de valoración y reflexión.

8. Implantación de ECTS en la materia de "Sociología de la Educación". En la asignatura de "Sociología de la Educación", el autor nos presenta cómo ha sido el diseño y desarrollo de la experimentación. Igual que en capítulos anteriores, inicia su trabajo haciendo una contextualización de ésta dentro del plan de estudios de la titulación; presenta el perfil que posee la materia; indica los objetivos que se persiguen y las competencias académicas y profesionales que se necesitan para el alcance de los mismos; explica el diseño de la adaptación mediante las metodologías adoptadas por el docente, las estrategias de aprendizaje que se han tenido en cuenta y el trabajo del alumnado que han desarrollado durante el curso de la materia. El autor también indica la cuantificación del trabajo de los alumnos y los métodos de evaluación seguidos; hace un análisis de los resultados académicos mediante la presentación y comentario de gráficos que versan 
sobre los textos utilizados, la evaluación continua seguida, la memoria de investigación que sus alumnos han realizado, la evaluación entre grupos, la memoria de la materia y la materia a nivel glogal. Termina su aportación, comunicando los resultados del proceso de evaluación realizado desde el punto de vista de su alumnado y desde su punto de vista como docente.

9. Valoración de la adaptación de la materia de "Desarrollo psicomotor" a los créditos ECTS en la titulación de Educación Infantil. Los dos autores de este octavo capítulo, presentan el resultado de la segunda fase del proyecto "Realización de estudios para la adaptación al Espacio Europeo en Titulaciones del Sistema Universitarios de Galicia", con la ampliación de las experiencias piloto en la titulación de Educación Infantil en la que imparten la materia de "Desarrollo psicomotor".

Con una línea de redacción similar a los anteriores capítulos ya presentados, estos docentes también comienzan explicando cómo ha sido el desarrollo de su experiencia. Así, hacen la descripción de la materia dentro del plan de estudios, señalan los descriptores de la misma, especifican datos sobre la matrícula y sobre el rendimiento académico de los alumnos en la materia en los últimos cuatro años.

A continuación, indican que el diseño de la adaptación está orientado por ocho objetivos y en una serie de competencias académicas y profesionales. Esto les permite referirse a la metodología docente empleada tanto en los créditos teóricos como en los prácticos (expositiva y activa por grupos e individual), a los medios didácticos utilizados (actividades y lecturas recomendadas) y a las modalidades formativas que se han tenido (sesiones presenciales, trabajo autónomo, trabajos dirigidos y tutorías).

A la luz de este diseño, los autores también presentan una serie de cronogramas que sirven para cuantificar el trabajo del docente y de los alumnos: uno genérico, otro de las clases eóricas y otro de las clases prácticas; se centran en distintos momentos de evaluación de los aprendizajes con sus respectivas técnicas; exponen los resultados académicos finales de su alumnado; presentan el instrumento utilizado para constatar el nivel de esfuerzo y opinión de los alumnos; y finalmente terminan realizando una valoración global de la experiencia en base a aspectos de organización de centros (calendario académico; proceso de matrícula; programa de convocatoria de exámenes; tratamiento de alumnos repetidores; organización horario de centros y titulaciones; reparto de espacios de trabajo común para el alumnado dentro del centro; y coordinación entre el profesorado involucrado en la experimentación con crédito ECTS y el que sigue con el antiguo sistema de enseñanzaaprendizaje); en base a aspectos docentes y académicos (dimensión práctica del currículo; planificación de la docencia; objetivos, contenidos y actividades de aprendizaje; metodología y recursos empleados; apoyo 
tutorial; mecanismos de evaluación del aprendizaje y reformulación de la propia evaluación; y ventajas y dificultades del nuevo sistema); y en función de la experimentación en términos generales.

10. Evaluación global del proceso de experimentación. El entonces decano de la Facultad de Ciencias de la Educación del campus de Ourense que ha coordinado el proceso de implementación de créditos ECTS en la titulación, cierra esta publicación con un aplauso a la experiencia llevada a cabo. Así, destaca las ventajas que supuso para la titulación, reconoce las dificultades encontradas y especifica cuestiones a tener en cuenta para el futuro.

\subsection{Titulación de Educación Infantil de Pontevedra}

En el campus de Pontevedra de la Universidad de Vigo también se imparte Educación Infantil, por ello la ACSUG les ha pedido su colaboración en esta tarea de experimentación ${ }^{6}$.

Como en otros volúmenes, los profesores implicados en la implantación de créditos ECTS, presentan su experiencia siguiendo un esquema similar al de la titulación de Psicología o al de la titulación de Actividad Física y del Deporte. Así, este volumen consta de 7 puntos que se describen a continuación.

El primero se corresponde con la introducción en la que se sintetizan los inicios del proyecto.

El segundo presenta el diseño del proyecto de adaptación indicando los objetivos a conseguir, las acciones que se derivan de los mismos y se caracteriza el grupo de trabajo.

El tercero explica el desarrollo de la experiencia según el tipo de actividades que se fueron realizando, por ejemplo, reuniones periódicas, elaboración de guías, fichas, entre otras.

En cuarto lugar, los autores realizan una valoración del trabajo del alumnado mediante la aplicación de determinados instrumentos $y$, los datos obtenidos, los sintetizan de acuerdo a las materias adaptadas.

En el quinto punto, se hace una evaluación glotal del proceso teniendo en cuenta el punto de vista del equipo decanal, profesorado implicado según su experiencia a nivel de materia, profesorado no implicado, alumnado y PAS.

Haciendo una síntesis de las valoraciones realizadas por los colectivos anteriores, en el sexto apartado se exponen una serie de recomendaciones para continuar con el proyecto en el futuro.

Por último, esta obra termina presentando los apéndices electrónicos de referencia en esta experiencia: las guías docentes de las materias adaptadas y otros documentos. 


\subsection{Titulación de Educación Infantil de grupo de Didácticas Especiales y adjuntos}

El trabajo de experimentación de créditos europeos de un pequeño grupo de profesores de las tres universidades gallegas toma cuerpo en el siguiente volumen ${ }^{7}$.

El presente volumen acoge la experiencia desde las Facultades de Ciencias de la Educación de las tres universidades gallegas. Como en otras ocasiones, tras una pequeña introducción y presentación del ejemplar, la obra se inicia con un primer punto que responde a los resultados y evaluación de la experiencia. En este momento, los autores especifican muy minuciosamente cómo se ha iniciado y desarrollado el proceso, las circunstancias particulares en que se estaba desarrollando; la documentación que se ha revisado.

En un segundo apartado de la publicación, se presentan las guías docentes de las siguientes materias adaptadas en los cursos 2004-05 y 2005-06: "Desarrollo de la expresión plástica y su didáctica" (USC), "Desarrollo psicomotor" (USC), "Lengua y literatura gallega" (USC), "Introducción al conocimiento matemático" (UDC) y "Desarrollo psicomotor" (UVIGO).

Finalmente, el informe termina con una evaluación del proceso desarrollado. Como viene siendo habitual, se presentan las valoraciones desde dos puntos de vista: el profesorado y el alumnado implicado. Sólo indicar que de los primeros se presenta su opinión sobre aspectos de organización de centros (calendario académico, proceso de matrícula, organización horaria de centros y titulaciones, coordinación entre profesorado, etc.), sobre aspectos docentes y académicos (dimensión práctica del currículo, apoyo tutorial, etc.) y sobre la experimentación en general; de los segundos, sólo se presentan unas reflexiones genéricas.

\section{A modo de conclusión}

A la luz de las publicaciones vistas, vemos que se trata de un conjunto de trabajos realizados por diferentes profesionales en los que se percibe la preocupación por caminar a la par de la convergencia europea desde un acto de voluntarismo. Asimismo, es de destacar el compromiso mantenido de cada uno de los profesionales que han participado y contribuido con sus dedicaciones y aportaciones más singulares. Estos hechos han servido para dar forma a experiencias muy singulares. Creemos firmemente que son necesarias publicaciones de este tipo que aborden el proceso de diseño y desarrollo de la experimentación de las materias de las titulaciones. Por lo tanto, además de constituir estos ejemplares herramientas útiles para el diseño de los futuros planos de estudio adaptados a las directrices del EEES, sólo resta felicitar a cada uno de los participantes y a la ACSUG por el gran valor didáctico de estas publicaciones. 


\section{Notas}

${ }^{1}$ Conferencia Mundial sobre Educación Superior (CMES) que desde 1998 impulsa el debate sobre las políticas de educación superior en el mundo.

${ }^{2}$ Véase en http://www.aneca.es/quees/queesfunciones.asp

3 El grupo de trabajo de la titulación de "Actividade Física e do Deporte" de la Universidad de Vigo estaba constituido por: José $\mathrm{M}^{a}$ Cancela Carral, Luis Casais Martínez, Gustavo Fuentes, Águeda Gutiérrez Sánchez, Alfonso Gutiérrez Santiago, $\mathrm{M}^{a}$ Carmen Iglesias Pérez, Cristina López Villar, Ana Isabel Rey Cao, Borja Novoa Castro y Juan Vidal Puga.

${ }^{4}$ El grupo de trabajo de la titulación de "Psicología" de la Universidad de Santiago estaba constituido por: Carolina Tinajero Vacas, $\mathrm{M}^{\mathrm{a}}$ José Ferraces Otero, Gloria Seoane Pesqueira, Socorro Rodríguez Holguín, Mauro Rodríguez Casal, Fernando Díaz Fernández y Susana Martínez Gomila.

${ }^{5}$ El grupo de trabajo de la titulación de "Educación Infantil" de la Universidad de Vigo (Campus Ourense) estaba constituido por: Xosé Manuel Cid Fernández, David Casado Neira, Javier García Núñez, María del Mar García Señorán, Josefina Pato Lorenzo, $M^{a}$ Esther Pérez Enríquez, Manuela Raposo Rivas, Domingo Rodríguez Teijeiro, Vicente Romo Pérez, Ma del Carmen Sarceda Gorgoso, Emilia Seoane Pérez y José Antonio Tarrío Fernández.

${ }^{6}$ El grupo de trabajo de la titulación de "Educación Infantil" de la Universidad de Vigo (Campus Pontevedra) estaba constituido por: Myriam Alvariñas Villaverde, Amada Traba Díaz y Jorge Soto Carballo.

7 El grupo de trabajo de la titulación de "Educación Infantil" de Grupo de Didácticas Especiais y adjuntos estaba constituido por: Carmen Franco Vázquez, Herminia García Ruso, Blanca-Ana Roig Rechou, Cristina Conde Pintos y Javier García Núñez.

\section{Referencias}

AA.VV. (2007). Titulación de Educación Infantil de Lugo. Proxecto ACSUG. Santiago de Compostela: ACSUG.

Benito, A. y Cruz, A. (2005). Nuevas claves para la docencia universitaria en el espacio europeo de educación superior. Madrid: Narcea.

Cancela Carral, J.M. y otros (2007). Titulación de Actividade Física e do Deporte. Proxecto ACSUG. Santiago de Compostela: ACSUG.

Cid Fernández, X.M. (coord) (2007). Titulación de Educación Infantil de Ourense. Proxecto ACSUG. Santiago de Compostela: ACSUG. 
Colás Bravo, C. y De Pablos, J. (coord.) (2005). La Universidad en la Unión Europea: el Espacio Europeo de Educación Superior y su impacto en la docencia. Aljibe. Málaga.

European Commission (2010). Focus in Higher Education in Europe 2010: The impact of the Bologna Process.- Brussels: Eusydice, 2010. Disponible en: http://eacea.ec.europa.eu/education/eurydice/documents/thematic reports/1 22EN.pdf (fecha consulta: 12.06.10).

Franco Vázquez, C. y otros (2007). Titulación de Educación Infantil de Grupo de Didácticas Especiais y adjuntos. Proxecto ACSUG. Santiago de Compostela: ACSUG.

García Manjón (coord.) (2009). Hacia el Espacio Europeo de Educación Superior: el reto de la adaptación de la Universidad a Bolonia. A Coruña: Netbiblo.

Martínez Figueira, M.E. (2008). Nivel de información del alumnado de Educación Social (Universidad de Vigo) sobre la adaptación a créditos europeos. Ponencia presentada en el Congreso Internacional: Comunicación, Calidad, Interculturalidad y Prospectiva en Educación. León, 6-9 mayo.

MCMES (2009). La Educación Superior en tiempos de cambio: nuevas dinámicas para la responsabilidad social: síntesis de los informes GUNI la Educación Superior en el mundo. Madrid: GUNI.

Tinajero Vacas, C. (2007). Titulación de Psicoloxía. Proxecto ACSUG. Santiago de Compostela: ACSUG.

Tinajero Vacas, C. (coord.) (2007). O traballo dos/as estudantes de Psicoloxía en termos ECTS. Proxecto ACSUG. Santiago de Compostela: ACSUG.

Villaverde, M. (coord.) (2007). Titulación de Educación Infantil de Pontevedra. Proxecto ACSUG. Santiago de Compostela: ACSUG.

\section{Nota Bibliográfica}

$M^{a}$ Esther Martínez Figueira es doctora en Ciencias de la Educación por la Universidad de Vigo. Su docencia es en la actualidad en Nuevas Tecnologías aplicadas a la Educación y Educación Ambiental. Por otra parte, sus líneas de investigación son en nuevas tecnologías, en docencia universitaria, en educación inclusiva así como en rúbricas y e-portfolio aplicado a la docencia.

Correspondencia con la autora: Despacho 26, planta baja, edificio 2.; Facultad Ciencias de la Educación (Campus Ourense). Universidad de Vigo. As Lagoas, s/n. CP 32004. Ourense; correo electrónico: esthermf@uvigo.es 